\section{Bio Core}

Exploring Scientific Community

\section{International Journal of Case Studies in Clinical}

\section{Research}

Case Report

ISSN 2572-102X

\title{
ECG in a Patient with a Fractured Arm Mimicking Atrial Flutter
}

Dakshin Gangadharamurthy ${ }^{1 *}$, Madhab Ray ${ }^{2}$

${ }^{1}$ Department of Internal Medicine, Brockton Hospital/Beth Israel Deaconess Medical Center, Brockton, MA

${ }^{2}$ Department of Internal Medicine, Brockton Hospital, Brockton, MA

${ }^{*}$ Corresponding Author: Dakshin Gangadharamurthy, MD, MPH, Department of Internal Medicine, Brockton Hospital/Beth Israel Deaconess Medical Center, Brockton, MA, USA, E mail: dakshin5131@yahoo.com

Citation: Dakshin Gangadharamurthy et al, (2017) ECG in a Patient with a Fractured Arm Mimicking Atrial Flutter. Int J clinical \& case. $1: 8,169-171$

Copyright: (C) 2017 Dakshin Gangadharamurthy et al. This is an open-access article distributed under the terms of the Creative Commons Attribution License, which permits unrestricted use, distribution, and reproduction in any medium, provided the original author and source are credited.

Received: November 01,2017; Accepted: November 13,2017; Published:November 30, 2017

Abstract

We present a patient with fracture of the right arm who had an electrocardiogram (ECG) that mimicked atrial flutter. We describe ways to correctly identify the rhythm in cases of suspected atrial flutter and describe the mechanism of the artifactual waves seen in our patient's ECG. Our case report also discusses specific ECG patterns that can be used to localize the source of the artifacts.

Keywords: ECG, Arrhythmia, Artifacts, Fracture

\section{Introduction}

Certain artifacts on ECG can mimic arrhythmias. The identification of artifacts is critical to avoid erroneous diagnoses, unwarranted anxiety, unnecessary diagnostic and therapeutic interventions, and potentially serious medical errors such as the initiation of antiarrhythmic therapy or long-term anticoagulation.

\section{Case Presentation}

A 95-year-old female presented to the emergency department complaining of right arm pain after a fall without loss of consciousness. She had no past medical history of cardiovascular disease. She denied having chest pain, palpitations, or light-headedness. On physical exam, she had a BP of 138/90, tachycardia and tachypnea. Her right arm was erythematous, swollen and tender to palpation associated with decreased range of motion. Radiography identified a closed fracture of the right humerus. Physical exam of rest of the systems was unremarkable.

\section{Investigations}

To investigate the tachycardia, a 12-lead ECG (Figure 1) was obtained, which, at first glance, revealed saw tooth-pattern waves suggestive of atrial flutter. However, on closer inspection, these waves were not seen in lead III, where $p$ waves could be seen prior to every QRS complex, establishing that the patient was, in fact, in sinus rhythm. The saw tooth waves, therefore, were artifactual or "pseudo-atrial flutter waves". Additional clues that helped confirm this were the irregular and varying morphology of the saw tooth waves, the lack of relationship between the waves and the QRS complexes, and the occurrence of the waves simultaneous with some of the QRS complexes. The sparing of lead III indicated that her left arm and lower limb electrodes were not involved in the cause of the artifact. Therefore, the artifact was related to her right limb electrode, which contributes to the tracings from all limb leads and precordial leads, with the exception of lead III. Correlating clinically, the patient was shaking her right arm in a tremor-like pattern due to pain from her right humeral fracture. 


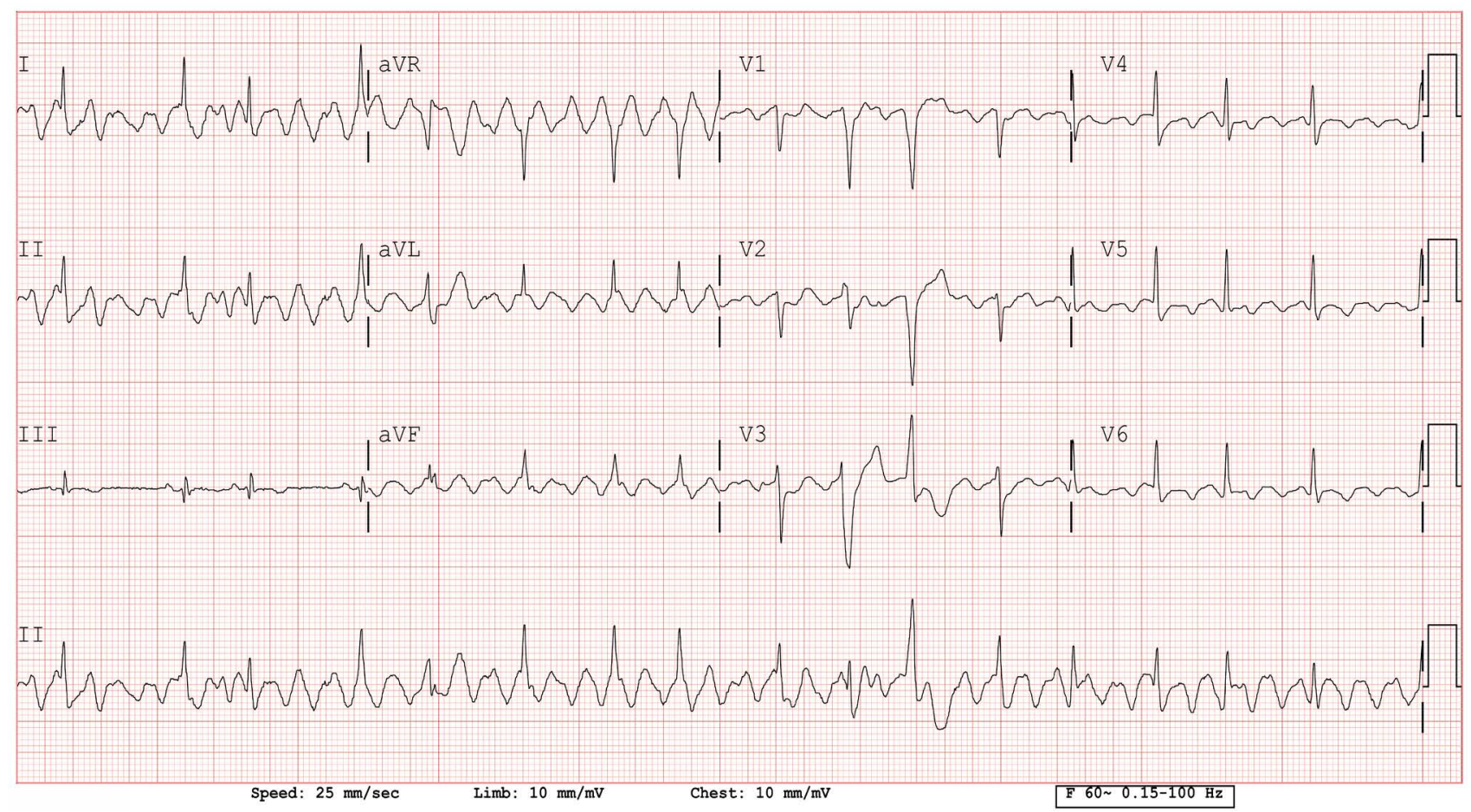

Figure 1: The ECG on presentation - what is the rhythm?

\section{Outcome And Follow-Up}

In our patient, a second ECG (Figure 2) was obtained after administration of pain medications and immobilization of the right arm, which showed sinus tachycardia with a ventricular pre- mature complex and absence of saw tooth waves. The patient's humerus fracture was managed conservatively with coaptation splint immobilization and pain control.

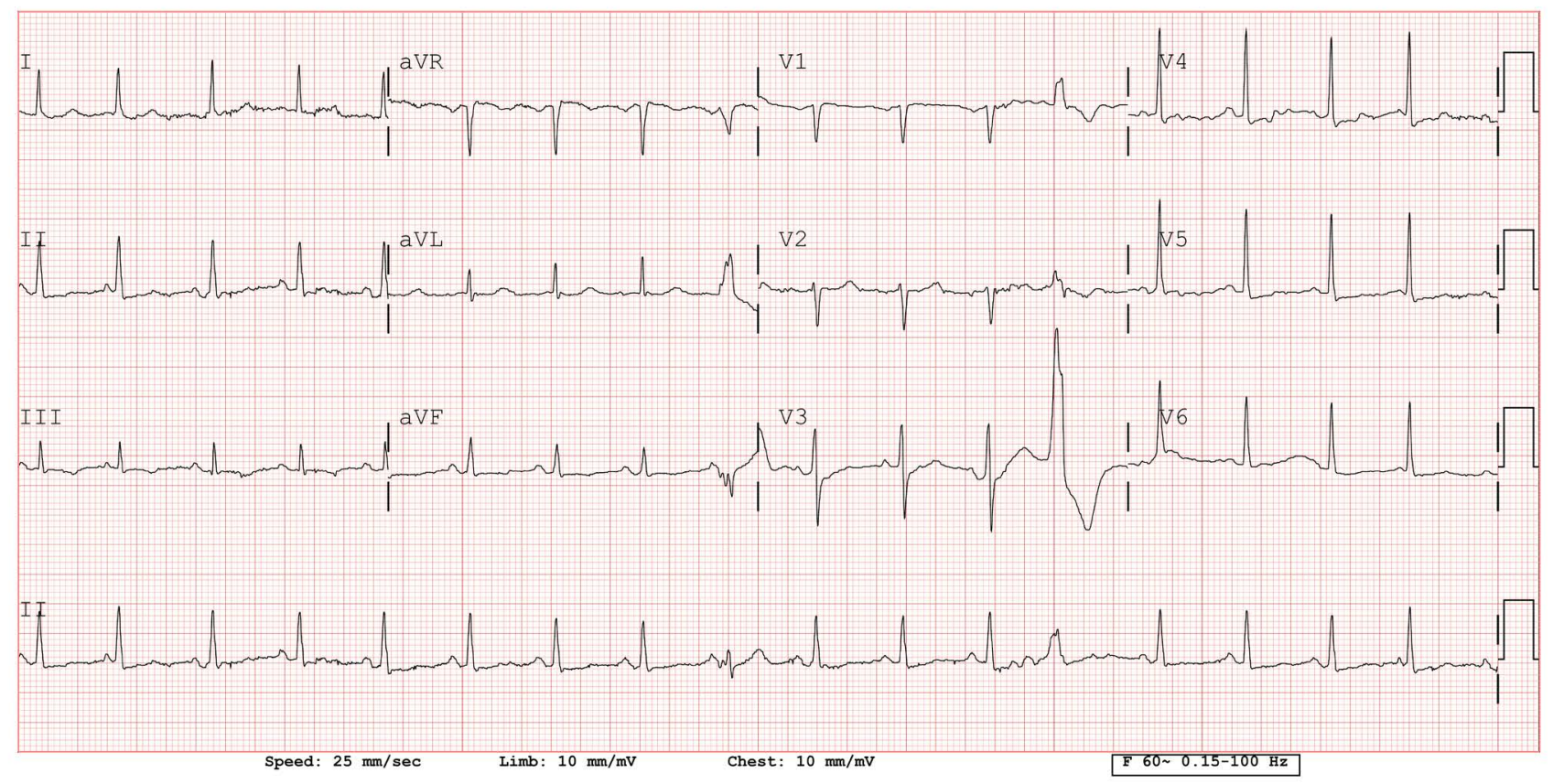

Figure 2: The second ECG after administration of pain medications and immobilization of the right arm 


\section{Discussion}

Artifacts mimicking atrial flutter and other arrhythmias have been described secondary to physiologic factors such as shivering from cold, Parkinsonian or benign essential tremor, hiccups, clonic contractions, poor electrode contact or other patient motion, or from non-physiologic factors such as electroconvulsive therapy, electromagnetic interference from electrical or electronic devices, or from devices such as left ventricular assist devices, continuous venovenous hemofiltration systems, implanted (deep thalamic, spinal cord) electro stimulators, transcutaneous electrical nerve stimulators, bladder stimulators, etc ${ }^{[1-7]}$. Pseudo-atrial flutter very similar to our patient's ECG has also been reported from a portable compact disc player placed close to the patient's right arm ECG lead ${ }^{[8]}$.

Inaccurate interpretation of ECGs due to artifacts may result in unwarranted anxiety, unnecessary diagnostic and therapeutic interventions, and potentially serious medical errors such as the initiation of antiarrhythmic therapy, or long-term anticoagulation for presumed atrial fibrillation or atrial flutter ${ }^{[9]}$. Therefore, identification of ECG artifacts is essential to avoid erroneous diagnoses. Recognizing certain ECG artifact patterns can assist in identifying the source of artifacts. For instance, with an ECG showing sparing of lead I from artifact, the source of the artifact would be the left leg. Similarly, sparing of lead II from artifact would suggest the left arm as the source of the artifact and sparing of lead III, as noted in our patient, would suggest the right arm as the source of the artifact ${ }^{[10]}$.

\section{Conclusion}

- ECG artifacts can mimic atrial flutter and other arrhythmias

- Inaccurate interpretation of ECGs due to artifacts may result in unwarranted anxiety, unnecessary or harmful interventions

- Recognizing certain ECG artifact patterns can assist in identifying and localizing the source of artifacts.

\section{References}

1. Patel SI, Souter MJ. Equipment-related electrocardiographic artifacts: causes, characteristics, consequences, and correction. Anesthesiology. 2008;108(1):138-48.

2. Saint-Pierre A. ECG artifacts simulating atrial flutter. JAMA: The Journal of the American Medical Association. 1973;224(11):1534.

3. Barold SS, Maloney JD, Herweg B. Diagnostic challenge of artifactual electrocardiographic tachyarrhythmias. Cardiol J. 2013;20(1):106-9. doi: 10.5603/CJ.2013.0019. PubMed PMID: 23558821

4. Baranchuk A, Kang J. Pseudo-atrial flutter: Parkinson tremor. Cardiol J, 2009; 16: 373-374.

5. Kostis WJ, Cohen L, Dominiecki SM. Continuous veno-venous hemodialysis pseudoflutter. J Electrocardiol. 2007 Oct;40(4):316-8. Epub 2006 Oct 25. PubMed PMID: 17069839

6. Khan IA. Differential electrocardiographic artifact from implanted thalamic stimulator. Int J Cardiol. 2004 Aug;96(2):285-6. PubMed PMID: 15262047

7. Patel SI. Electrocardiographic artifacts during electroconvulsive therapy. J Electrocardiol. 2009 Jul-Aug;42(4):307-9. doi: 10.1016/j.jelectrocard.2009.02.003. Epub 2009 Apr 2. PubMed PMID: 19344913

8. Austin SM, Flach SD, Gaines CM. Atrial flutter simulated by a portable compact disk player. Mayo Clinic Proceedings. 2007;82(3):383-4.

9. Bogun, F., Anh, D., Kalahasty, G. et al, Misdiagnosis of atrial fibrillation and its clinical consequences. Am J Med. 2004;706

10. Rowlands DJ, Moore PR. Spurious atrial tachycardia in a pilot. Prediction of the artifactual appearances in all twelve leads, when a single limb lead is the source of the artifact. Journal of Electrocardiology. 2007;40(6):475-7. 